\title{
The scheduling with rejection on uniform multiprocessors
}

\author{
Liu Shoupeng ${ }^{1, a^{*}, \text { Wei fei }}{ }^{2, b}$ \\ ${ }^{1}$ Bin Zhou Medical College, Yantai, Shandong, China,264003 \\ ${ }^{2}$ Bin Zhou Medical College, Yantai, Shandong, China,264003 \\ aliushoupeng@eyou.com, ${ }^{\mathrm{a}}$ peterfei3000@163.com
}

Keywords: On-line scheduling; Multiprocessors; The competitive ratio; Rejection; Algorithm.

\begin{abstract}
Objective To propose an approximate for the scheduling with rejection on uniform machines. Methods Design an on-line algorithm. Results We prove the competitive ratio of the algorithm. Conclusion The competitive ratio of the algorithm does not beyond $1+\beta$.
\end{abstract}

\section{Introduction}

In classical deterministic scheduling problems, it is assumed that all jobs have to be processed. However, in many practical cases, mostly in highly loaded make-to-order production systems, accepting all jobs may cause a delay in the completion of orders which in turn may lead to high inventory and tardiness costs. Thus, in such systems, the firm may wish to reject the processing of some jobs by either outsourcing them or rejecting them altogether. The field of scheduling with rejection provides schemes for coordinated sales and production decisions by grouping them into a single model. Since scheduling problems with rejection are very interesting both from a practical and a theoretical point of view, they have received a great deal of attention from researchers over the last decade.

In this paper, we consider an on-line scheduling problem with rejection .The job can be processed, or be rejected, but to pay a certain penalties. It may be stated as follows: the model have $\mathrm{n}$-independent, non-preemptive jobs, $\mathrm{J}=\left\{\mathrm{J}_{1}, \mathrm{~J}_{2}, \ldots, \mathrm{J}_{\mathrm{n}}\right\}$, are available for processing at time zero on a set of $m$ machines $M=\left\{M_{1}, M_{2}, \ldots, M_{m}\right\}$ arranged according to a specific and predefined machine environment. In this paper, we consider the uniform machines. Each processor in a uniform multiprocessor machine is characterized by a speed $s_{i}(i=1,2, \cdots, m)$, Might as well set $s_{1} \geq s_{2} \geq \cdots \geq s_{m}=1$. The input for a problem of scheduling with rejection includes the following parameters: $p_{j}$, which is the processing time of job $J_{j}(\mathrm{j}=1, \ldots, \mathrm{n})$ and $r_{j}$ which is the rejection cost of job $J_{j}$. The processing time of job $J_{j}$ that are processed on machine $\mathrm{M}_{\mathrm{i}}(\mathrm{i}=$ $1, \ldots, \mathrm{m})$ is $p_{j} / s_{i}$. For set $S \subseteq J, R(S)$ is the total rejected cost in $\mathrm{S}$, namely $R(S)=\sum_{j \in S} r_{j}$.

Given a solution produced by the on-line algorithm SRUM (scheduling with rejection on uniform multiprocessors), A refers to the set of accepted jobs, and set $\mathrm{R}$ refers to the set of rejected jobs. 
$p_{\max }$ denotes the largest processing time of all accepted jobs. For their analogs in the optimal solutions we use $\bar{R}, \bar{A}, \quad \bar{p}_{\max }$, respectively. $Z^{A_{1}}$ denotes the total cost of the Algorithm SRUM and $Z^{\text {opt }}$ is the cost achieved by the optimal solution. In the following proof, we make the $M(S)=\sum_{j \in S} \frac{p_{j}}{\sum s_{i}}$.The set $B=\left\{j \mid r_{j} \leq \frac{p_{j}}{\sum s_{i}}\right\}$ contains jobs with penalty less than or equal to their load.

The off-line scheduling with rejection penalty is NP-hard. It does not exist a polynomial time optimal algorithm. Therefore, we propose an approximate Algorithm SRUM. It rejects all job from set $B=\left\{j \mid r_{j} \leq \frac{p_{j}}{\sum s_{i}}\right\}$, and accepts part of the jobs in the rest of the jobs. Next, we propose an on-line algorithm SRUM and analysis the competitive ratio of it .

\section{Algorithm SRUM:}

Step 1: If a job $J_{i}$ from $B$ is arrive, reject it;

Step 2: Let $P$ be the total penalty of all rejected jobs from $J-B$.If the job $\mathrm{J}_{\mathrm{j}}=\left(\mathrm{t}_{\mathrm{j}}, \mathrm{p}_{\mathrm{j}}\right) \notin B$ is arrive, reject it if $P+r_{j} \leq \alpha p_{j}$, otherwise, accept it and schedule it on a least loaded machine by LS algorithm.

On the Algorithm SRUM, Let $\alpha=\frac{\sqrt{5-1}}{2}, \varphi=\frac{\sqrt{5+1}}{2}$, and c satisfy

$$
c \geq 1+\left(\frac{1}{s_{m}}-\frac{1}{\sum_{i=1}^{m} s_{i}}\right) \frac{1}{\alpha}, \quad c \geq 2+\alpha \frac{2}{\sum_{i=1}^{m} s_{i}}
$$

Theorem: The Algorithm SRUM from $m$ machines is $1+\beta$ competitive ,

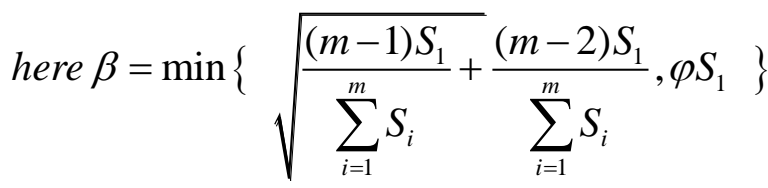

Proof: First for certain optimal solution, we define $S=R \cap \bar{A}-B$ as job set, it satisfy in the optimal solution be accepted but in step 2 rejected, The completion of the machine is the first $\mathrm{k}$ machine $M_{k}$, Maximum hours of accepting jobs is $p_{\max }$, the processing time of the last job is $p_{l}$, By the algorithm LS, we obtain 


$$
\begin{gathered}
C_{k}+\frac{p_{l}}{S_{k}} \leq C_{i}+\frac{p_{l}}{S_{i}},(i=1,2, \cdots m) \\
C_{k}+\frac{p_{l}}{S_{k}} \leq C_{\min }+\frac{p_{l}}{S_{m}} \leq C_{\text {min }}+p_{\text {max }},
\end{gathered}
$$

By the formula (1) and $p_{l} \leq p_{\max }$,we can obtain

$$
C_{k}+\frac{p_{l}}{S_{k}} \leq M(A)+\frac{(m-1) p_{l}}{\sum_{i=1}^{m} S_{i}} \leq M(A)+\frac{(m-1) p_{\mathrm{max}}}{\sum_{i=1}^{m} S_{i}}
$$

Then

$$
\begin{aligned}
& Z^{o n}=C_{k}+\frac{p_{l}}{C_{k}}+P(R) \leq M A+\frac{\left(m-1 p_{\mathrm{max}}\right.}{\sum_{i=1}^{m} S_{i}}+P(R) \\
& =\left(M(A+P R-S+) M S(+)^{(m-1)} \frac{1 \phi_{\mathrm{max}}}{\sum_{i=1}^{m} S_{i}}+P(S)-M \$\right.
\end{aligned}
$$

We first consider the left half part

$$
(M(A+P(R \quad S) M(S
$$

$M\left(A \bigcap^{-} A\right)+M\left(A^{-} \mathbb{R} \quad P\left(-R \cap \bar{S} \quad \forall \quad P\left(\mathbb{R}^{-} S+\mathbb{R}\right.\right.\right.$

$\leq M(\bar{A})+P \overline{(} R) \leq{ }^{o} Z^{T}$

Since all jobs in $\mathrm{S}$ are accepted by the optimal solutions, So the largest processing time is not more than $\bar{p}_{\text {max }}$. By the algorithm, we can obtain $P(S) \leq \alpha \bar{p}_{\text {max }}$. For any rejected job $\mathrm{J}$ by step 2, we know $r_{j} \leq \alpha p_{j}$, Summation on both sides, we can obtain $P(S) \leq \alpha M(S) \sum_{i=1}^{m} S_{i}$.So

$$
P(S)-M(S) \leq\left(1-\frac{1}{\alpha \sum_{i=1}^{m} S_{i}}\right) P(S) \leq\left(1-\frac{1}{\alpha \sum_{i=1}^{m} S_{i}}\right) \alpha \bar{p}_{\text {max }}=\left(\alpha-\frac{1}{\sum_{i=1}^{m} S_{i}}\right) \bar{p}_{\text {max }}
$$

1) If $p_{\max } \leq \bar{p}_{\max }$, then $P(S)-M(S)+\frac{(m-1) p_{\max }}{\sum_{i=1}^{m} S_{i}} \leq\left(\alpha-\frac{m-2}{\sum_{i=1}^{m} S_{i}}\right) \bar{p}_{\text {max }}$

2) If $p_{\max } \geq \bar{p}_{\max }$, Let $P$ be the total penalty incurred by the jobs in Step 2 of the algorithm until it schedules the first job with processing time is $p_{\max }$. 
By the condition Step 2 of the algorithm, $\alpha p_{j} \leq P+r_{j}$.On the other hand $P+r_{j} \leq P(S)+P(\bar{R})$, as all rejected jobs in Step 2 are in $S \cup \bar{R}$, and also the job j with processing time is in $\bar{R}$, since $p_{\max } \geq \bar{p}_{\max }$. Thus

$$
\begin{aligned}
& P(S)-M(S)+\frac{(m-1) p_{\mathrm{m} \mathrm{a}}}{\sum_{i=1}^{m} S_{i}} \leq\left(\alpha-\frac{1}{\sum_{i=1}^{m} S_{i}}\right) \bar{p}_{\mathrm{m} \mathrm{a}}+\frac{m-1}{\sum_{i=1}^{m} S_{i}} \cdot \frac{1}{\alpha}\left(P+r_{j}\right) \\
\leq & \left(\alpha-\frac{1}{\sum_{i=1}^{m} S_{i}}\right) \bar{p}_{\max }+\frac{m-1}{\sum_{i=1}^{m} S_{i}} \cdot \frac{1}{\alpha}(P(S)+P(\bar{R})) \\
\leq & \left(\alpha+\frac{m-2}{\sum_{i=1}^{m} S_{i}}\right) \bar{p}_{\max }+\frac{m-1}{\alpha \sum_{i=1}^{m} S_{i}} P(\bar{R})
\end{aligned}
$$

If $\quad \alpha=\sqrt{\frac{m-1}{S_{1} \sum_{i=1}^{m} S_{i}}}, \quad\left(\alpha+\frac{m-2}{\sum_{i=1}^{m} S_{i}}\right) \bar{p}_{\max }+\frac{m-1}{\alpha \sum_{i=1}^{m} S_{i}} P(\bar{R}) \quad$ achieve $\quad$ Minimum value , So

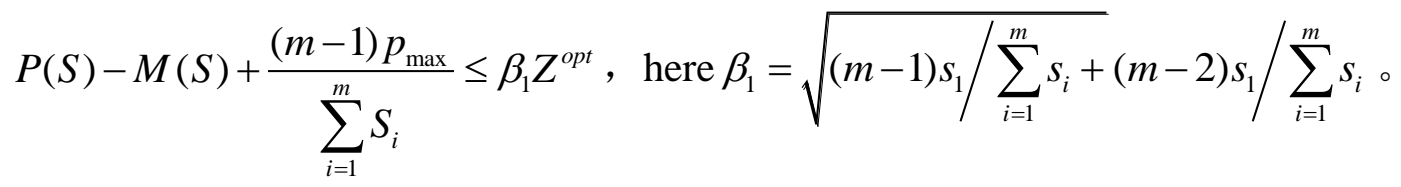

By Formula (2),we can obtain

$$
\begin{aligned}
& Z^{o n}=C_{k}+\frac{p_{l}}{S_{k}}+P(R) \leq C_{\min }+p_{\max }+P(R) \\
& \leq M(A)+P(R)+\left(\frac{1}{S_{m}}-\frac{1}{\sum_{i=1}^{m} S_{i}}\right) p_{\max } \\
& =(M(A)+P(R-S)+M(S))+P(S)-M(S)+\left(\frac{1}{S_{m}}-\frac{1}{\sum_{i=1}^{m} S_{i}}\right) p_{\max }
\end{aligned}
$$

From the above proof, we can obtain

$$
M(A)+P(R-S)+M(S) \leq M(\bar{A})+P(\bar{R}) \leq z^{\text {opt }}
$$




$$
P(S)-M(S)+\left(\frac{1}{S_{m}}-\frac{1}{\sum_{i=1}^{m} S_{i}}\right) T \leq\left(\alpha+\frac{1}{S_{m}}-\frac{2}{\sum_{i=1}^{m} S_{i}}\right) \bar{p}_{\max }+\left(\frac{1}{S_{m}}-\frac{1}{\sum_{i=1}^{m} S_{i}}\right) \frac{1}{\alpha} P(\bar{R})
$$

We know $\alpha=\varphi-1, c=\varphi+1$ and satisfy the following inequality

$$
c \geq 1+\left(\frac{1}{S_{m}} \frac{1}{\sum_{i=1}^{m} S_{i}}\right)^{\frac{1}{\alpha}}, \quad c \geq 2+\alpha \frac{2}{\sum_{i=1}^{m} S_{i}}
$$

So we have

$$
Z^{o n} \leq Z^{o p t}+(c-1)(P(\bar{R})+\bar{T})=Z^{o p t}+\varphi(P(\bar{R})+\bar{T}) \leq\left(1+\phi S_{1}\right) Z^{O P T}
$$

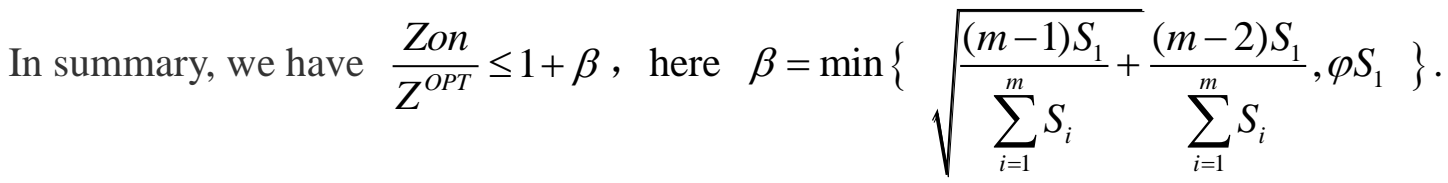

\section{The Conclusion}

In this paper, we propose an on line algorithm for the scheduling with rejection penalty and analysis the competitive ratio of it. It extend the results of Yair bartal to uniform machines. But some open problems remain. This requires that we continue to work hard.

\section{References}

[1] Bartal, Y., Leonardi, S., Marchetti-Spaccamela, A., Sgall, J., and Stougie, L., 2000, Multiprocessor Scheduling with Rejection, in: Seventh ACM-SIAM Symposium on Discrete Algorithms, 95-103.

[2] Cao, Z., Wang, Z., and Shoupeng, L., 2006, On Several Scheduling Problems with Rejection or Discretely Compressible Processing Times, in: Lecture notes in computer science, 3959, 90-98.

[3] Cao, Z., Yang, X., 2009, A PTAS for Parallel Batch Scheduling with Rejection and Dynamic Job Arrivals, Theoretical Computer Science, 410, 2732-2745.

[4] Cao, Z. and Zhang, Y., 2007, Scheduling with rejection and non-identical job arrivals, Journal of Systems Science and Complexity, 20, 529535.

[5] Cesaret, B., O־guz, C., \& Salman, F. S. (2012). A tabu search algo-rithm for order acceptance and scheduling. Computers and Opera-tions Research, 39(6), 1197-1205.

[6] Choi, B. C., \& Chung, J. (2011). Two-machne flow shop schedulng problem with an outsourcing option. European Journal of Opera-tional Research, 213, 66-72.

[7]Steiner, G., \& Zhang, R. (2011). Revised delivery-time quotation in scheduling with tardiness penalties. Operations Research, 59, 1504- 1511.

[8]Shabtay, D., Gaspar, N., \& Yedidsion, L. (2012). A bicriteria approach to scheduling a single machine with rejection and positional penalties. Journal of Combinatorial Optimization, 23(4), 
395-424.

[9]Shabtay, D., \& Gaspar, N. (2012). Two-machine flow-shop with rejec-tion. Computers and Operations Research, 39(5), 1087-1096.

[10] Zhang, L., Ren, J., and Wang, C., 2009, Scheduling with Rejection to Minimize the Makespan, in: Lecture notes in computer science, 5573, 411-420. 